\begin{tabular}{l}
\hline OPEN O ACCESS Freely available online \\
http://www.banglajol.info/index.php/BJID/index \\
Case Report \\
Bangladesh Journal of Infectious Diseases \\
December 2017, Volume 4, Number 2 \\
ISSN (Online) 2411-670X; ISSN (Print) 2411-4820 \\
DOI: http://dx.doi.org/10.3329/bjid.v4i2.37696
\end{tabular}

\title{
Cerebral Aspergillosis in an Immunocompetent Patient: A Case Report
}

\author{
Kaisar Haroon ${ }^{1}$, Tania Taher ${ }^{2}$, Abdullah Alamgir ${ }^{3}$, Md. Arif Reza ${ }^{4}$, Sabrina Sharmin ${ }^{5}$, Labiba Sharmin ${ }^{6}$, \\ Fatima Amin ${ }^{7}$, Sk. Sader Hossain ${ }^{8}$
}

\begin{abstract}
${ }^{1}$ Assistant Professor, Department of Neurosurgery, National Institute of Neurosciences \& Hospital, Dhaka, Bangladesh; ${ }^{2}$ Assistant Professor, Department of Paediatrics, MH Samorita Medical Hospital \& College, Dhaka, Bangladesh; ${ }^{3}$ Associate Professor, Department of Neurosurgery, National Institute of Neurosciences \& Hospital, Dhaka, Bangladesh; ${ }^{4}$ Medical Officer, Department of Neurosurgery, National Institute of Neurosciences \& Hospital, Dhaka, Bangladesh; ${ }^{5}$ Medical Officer, Department of Neurosurgery, National Institute of Neurosciences \& Hospital, Dhaka, Bangladesh; ${ }^{6}$ Honorary Medical Officer, Department of Neurosurgery, National Institute of Neurosciences \& Hospital, Dhaka, Bangladesh; ${ }^{7}$ Honorary Medical Officer, Department of Neurosurgery, National Institute of Neurosciences \& Hospital, Dhaka, Bangladesh; ${ }^{8}$ Professor \& Head, Department of Neurosurgery, National Institute of Neurosciences \& Hospital, Dhaka, Bangladesh
\end{abstract}

[Received: 1 October 2017; Accepted: 15 November 2017; Published: 1 December 2017]

\begin{abstract}
Aspergillus is a fungus found in the environment. In an immunocompetent person, inhalation of spores may cause localized infection. Invasive aspergillosis has a poor prognosis. We describe a case of cerebral aspergillosis in an immune competent patient. A 30-year-old man was admitted with seizures and headaches. Magnetic resonance imaging (MRI) of the brain showed contrast enhancing lesion at the suprasellar region. Excision biopsies showed granulomatous reactions, mixed inflammatory infiltration, fibrosis, and necro-purulent material mixed with fungal hyphae featuring acute-angle branching and septation, which was compatible with aspergillosis. Amphotericin B was begun. The results of testing for human immunodeficiency virus (HIV) was negative. Unfortunately the patient died on twentieth postoperative day. Most cases of invasive aspergillosis show that this organism is pathogenic in immunocompromized patients; however, some case reports show that invasive aspergillosis may not be so rare in immunocompetent patients. In these patients, virulent and drug-resistant forms of Aspergillus may be responsible for the disease, and treatment with antifungal agents is often ineffective, so that surgical excision is required. [Bangladesh Journal of Infectious Diseases 2017;4(2):52-55]
\end{abstract}

Keywords: Aspergillosis; brain abscess; meningitis; immunosuppression; immunocompetence; mycoses; voriconazole; amphotericin B

Correspondence: Dr. Kaisar Haroon, Assistant Professor, Department of Neurosurgery, National Institute of Neurosciences \& Hospital, Dhaka, Bangladesh; Email: kaisar298@ gmail.com; Cell no.: +8801711196577

Conflict of interest: There is no conflict of interest to any of the authors of this article.

Contribution to authors: Haroon K, Taher T, Alamgir A, Reza MA, Sharmin S contributed in patient management up to article write up. Sharmin L, Amin F, Hossain SS authors revised and corrected the paper.

How to cite this article: Haroon K, Taher T, Alamgir A, Reza MA, Sharmin S, Sharmin L, Amin F, Hossain SS. Cerebral Aspergillosis in an Immunocompetent Patient: A Case Report. Bangladesh J Infect Dis 2017;4(2):52-55

Copyright: @2017. Haroon et al. Published by Bangladesh Journal of Infectious Diseases. This article is published under the Creative Commons CC BY-NC License (https://creativecommons.org/licenses/by-nc/4.0/). This license permits use, distribution and reproduction in any medium, provided the original work is properly cited, and is not used for commercial purposes.

\section{Introduction}

Fungal infections of the CNS are challenging to treat and their optimal management requires knowledge of their epidemiology, host characteristics, diagnostic criteria, and therapeutic options. Aspergillus and Cryptococcus species predominate among fungal infections of the $\mathrm{CNS}^{1}$. 
Aspergillosis is a relatively infrequent opportunistic infection of the CNS that may account for around $10 \%$ of all fungal infections of the CNS and it is associated with high mortality and morbidity ${ }^{2}$. The involvement of the CNS may occur either by direct propagation from sinuses, nose or ear canal, or by hematogenous spread from a primary pulmonary focus ${ }^{2}$.

The most common port of entry is by inhalation of the spores, causing sino-pulmonary diseases. The other less common port of entry is direct implantation of spores when there is breach in mucocutaneous barriers as in burns and sites of catheter insertion or trauma ${ }^{3}$. According to JaeChang Lee, Central nervous system (CNS) Aspergillus abscess is very rare in immunocompetent patients and a potentially life threatening disease that is usually misdiagnosed because its presentation is similar to that of a tumor ${ }^{4}$. Invasive aspergillosis has a poor prognosis, particularly in critically ill patients with cerebral involvement. The mortality rate of cerebral aspergillosis $63 \%{ }^{5}$. According to the Infectious Diseases Society of America, Aspergillus species continue to be an important cause of life threatening infection. Voriconazole is recommended as primary therapy for CNS aspergillosis (strong recommendation; moderate-quality evidence). Lipid formulations of $\mathrm{AmB}$ are reserved for those intolerant or refractory to voriconazole ${ }^{6}$. Fasciano et $\mathrm{al}^{7}$ suggested Amphotericin B as the antifungal drug of choice for treatment of aspergillosis and can be delivered either intravenously or intrathecally.

\section{Case Presentation}

A thirty year old non diabetic, non-hypertensive man was admitted to the Department of Neurosurgery, National Institute of Neurosciences \& Hospital, Dhaka, Bangladesh hospital with the complaints of progressively severe headache, nausea, vomiting and deteriorating vision for four months.

Headache was gradually aggravating in the morning without any precipitating factors, but it was accompanied by nausea and vomiting. He never had a convulsion or lost consciousness. On examination, his higher psychic function was normal. His cranial nerves were intact except $2^{\text {nd }}$ cranial nerve. There was no perception of light or projection of rays. His pupils were bilaterally dilated. He had no motor or sensory deficit and also no signs of cerebellar dysfunction or meningeal irritation. All blood counts were normal.

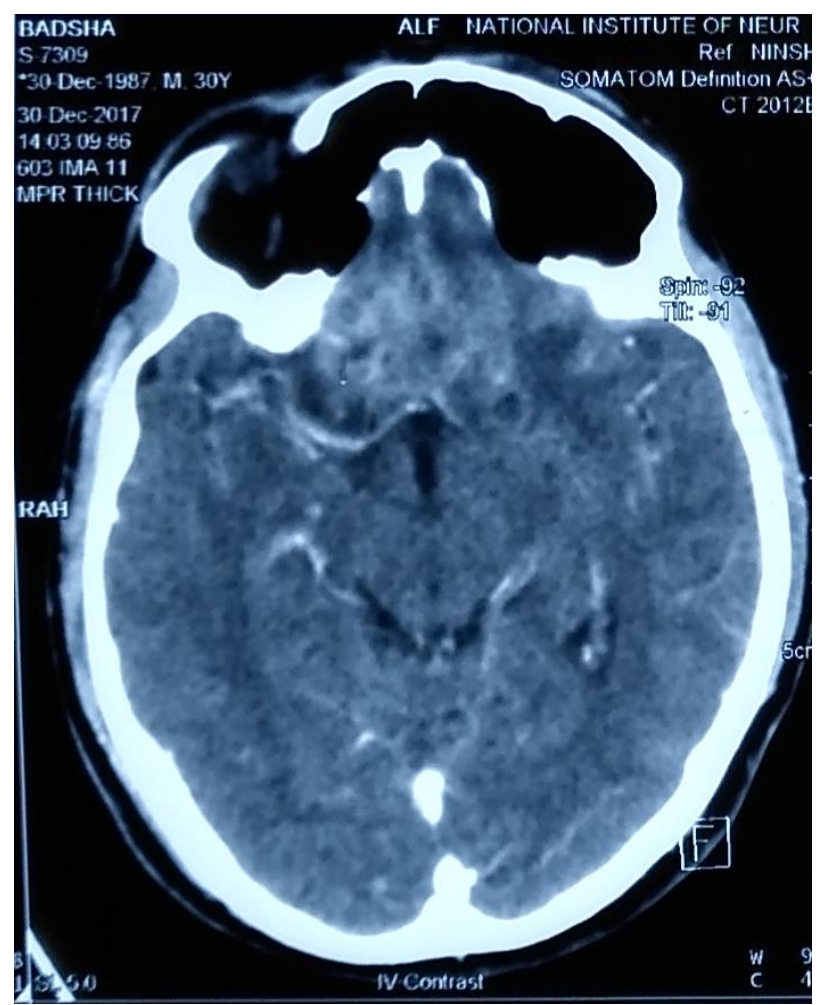

Figure 1: CECT Contrast enhancing lesion of Suprasellar region

The chest x-ray was normal. The serological test of the patient for HBV and HIV were negative. CT scan (Figure 1) showed a contrast enhancing lesion in the sellar and supra sellar region extending up to the tuberculum sella.

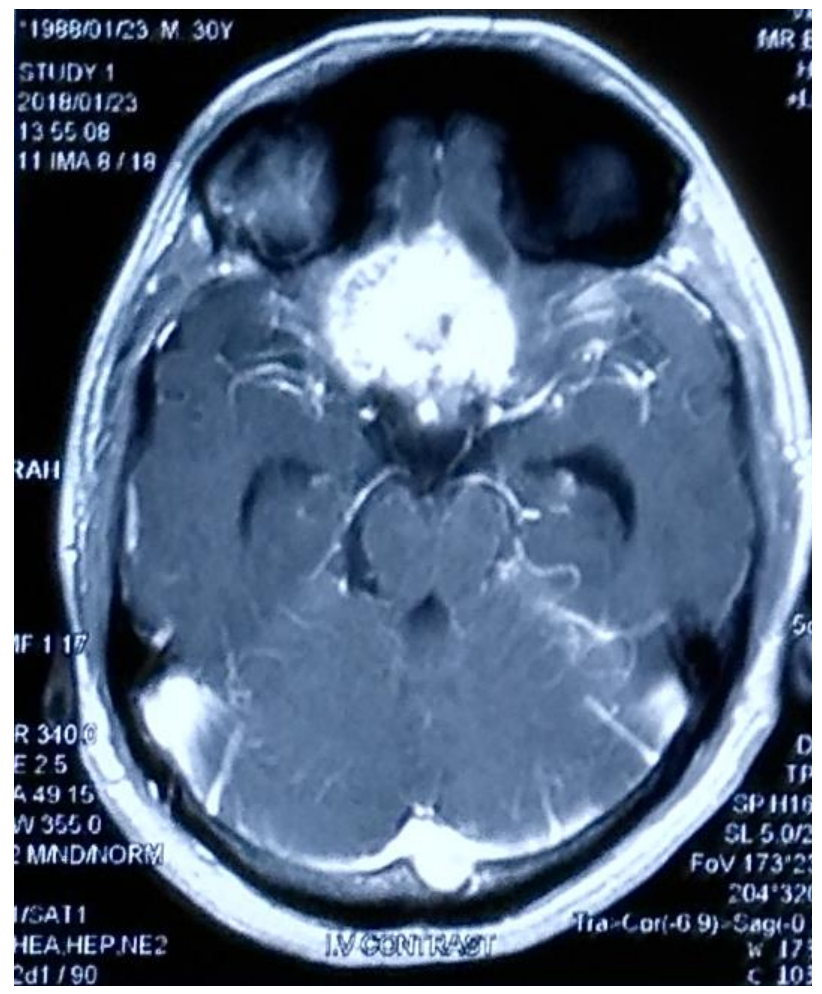

Figure 2: MRI of brain with contrast 


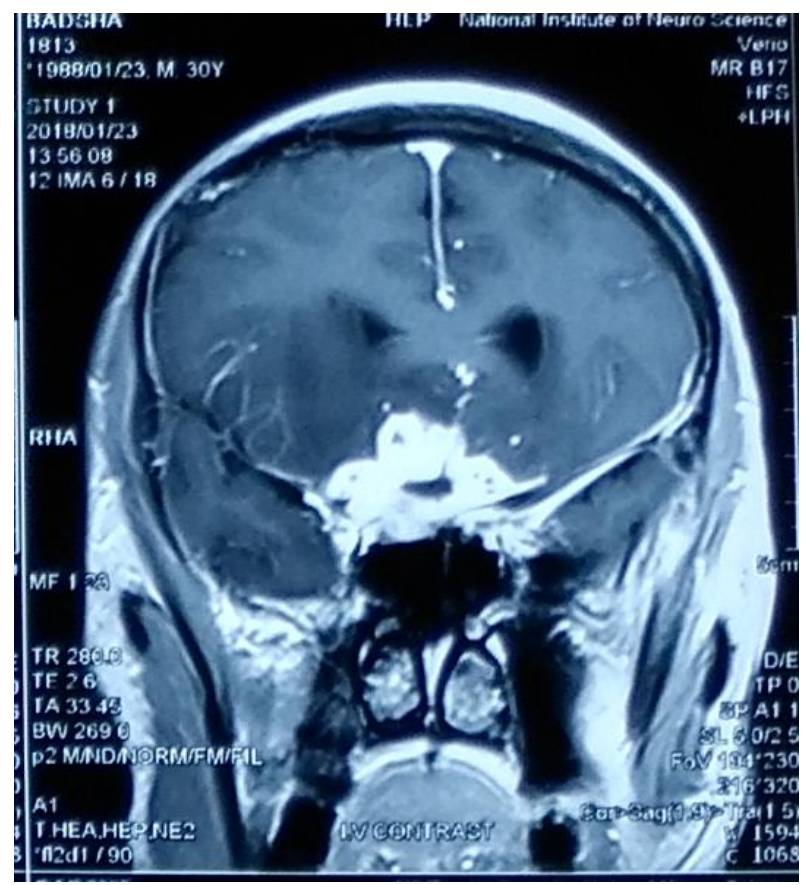

Figure 3: MRI of Brain with Contrast in Coronal Section

MRI of brain (Figure 2) showed a brilliant contrast enhancing lesion at the suprasellar and tuberculum sella attached with the basal dura. The patient was diagnosed as tuberculum sella meningioma. The differential diagnosis was supra sellar tuberculoma.

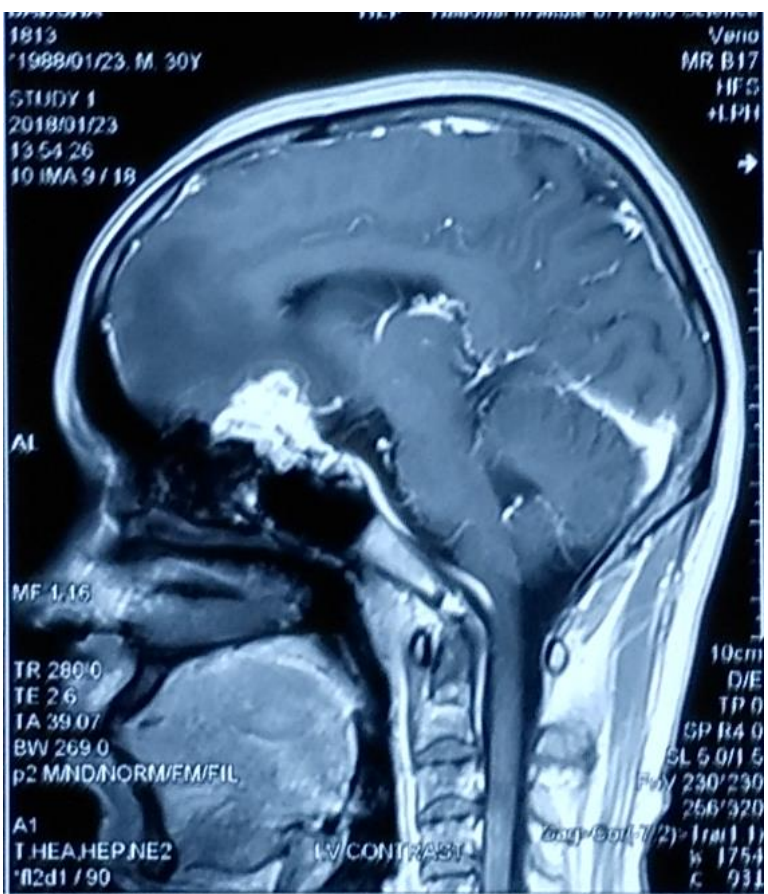

Figure 4: MRI of Brain with Contrast Sagittal Section

After admission to the hospital suddenly his headache and vomiting increased with deterioration of consciousness. The patient underwent emergency EVD following which his GCS had improved. Then definitive surgery was performed. The tumour was approached through a sub-frontal craniotomy. After retraction of the frontal lobe, the lesion was found to be firm to hard in consistency and grayish in colour. There was neither a cleavage plane nor any display of caseous material. It was moderately vascular. Severe adhesion with the surrounding tissue was encountered. Subtotal resection was done. Dura was closed water tight. Wound was closed in layers. The histopathological report revealed fungal colonies surrounded by many acute and chronic inflammatory cells. The fungi show narrow hyphae, branching at acute angles with terminal spore. Some of the fragments show glial tissue at the periphery. No evidence of tumour or malignancy was seen. Their comment was: fungal granuloma compatible with aspergillosis (Figure 3).

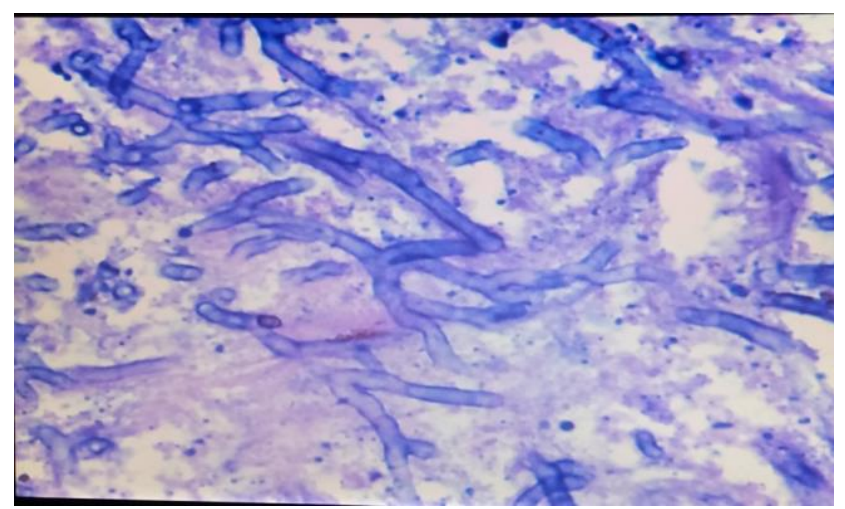

Figure 5: Fungal hyphae with spores on Histopathology (H\&E preparation)

Amphotericin B was started immediately following receipt of the histopathology report. At first, his condition remained unchanged and his stitches were taken off on the $9^{\text {th }}$ POD. After $14^{\text {th }}$ POD, his level of consciousness deteriorated gradually. Unfortunately, despite appropriate medical and surgical treatment, the disease progressed and the patient eventually died 20 days after surgery.

\section{Discussion}

CNS aspergillosis is rare. However, the number of reported cases increased significantly in the last decade. Aspergillus can reach the CNS by three different routes. The first is by hematogenous spread from a remote extracranial focus. The second is by extension from a contiguous extracranial location, and the third is direct introduction of Aspergillus by a neurosurgical procedure iatrogenically ${ }^{4}$. In immunocompetent patients, virulent and drug-resistant forms of Aspergillus may be responsible for the disease, and treatment with antifungal agents is often ineffective, so that surgical excision is required. 
In immunocompetent patients, this fungus spreads from sites near the brain, such as the sinuses ${ }^{5}$. Artico stated the factors positively influencing the effectiveness of therapy to be evidence of single, isolated lesion without dissemination; paucity of neurologic symptoms; early diagnosis; preventive amphotericin B administration in patients at risk for aspergillosis $^{8}$. In the brain, contiguous spread occurs from granulomatous lesions in the paranasal sinuses and middle ear leading to the development of chronic granuloma with dense fibrosis in adjacent structures of the brain, like the frontal lobe, from paranasal sinuses. Rhinocerebral granulomas occurs commonly in immunocompetent hosts. Rhinocerebral lesions are usually diagnosed early due to easier sampling from paranasal sinuses and have less mortality ${ }^{9}$. In this patient the lesion was adjacent to the paranasal sinuses. Administration of broad-spectrum antibiotics over a 3-month period has been shown to predispose to central nervous system aspergillosis ${ }^{10}$.

The treatment of choice for sino-orbital aspergillosis or sphenoidal is surgical excision of the aspergilloma, granulation tissue of the involved sinus for ventilation and drainage, as early as possible. Furthermore, there was shown a beneficial effect of pre-surgical treatment with antifungal therapy. In patients for whom total removal cannot be achieved, intensive therapy with antifungal agents must be started immediately ${ }^{11}$.

\section{Conclusion}

CNS aspergillosis is rare but very serious, with a high mortality. A combination of surgical resection and antifungal therapy does not guarantee a good outcome. The prognosis of the patients depends on early diagnosis and prompt aggressive treatment.
Therefore, suspicion of aspergillosis at nearby areas of paranasal sinuses should be borne in mind.

\section{References}

1. Schwartz S, Kontoyiannis DP, Harrison T, Ruhnke M. Advances in the diagnosis and treatment of fungal infections of the CNS. The Lancet Neurology. 2018 February 21, 2018(17):362-72

2. Carod-Artal FJ. Fungal Infections of the Central Nervous System. CNS Infections: Springer; 2018. p. 129-56

3. Challa S. Pathogenesis and Pathology of Invasive Aspergillosis. Current Fungal Infection Reports. 2018:110

4. Lee J-C, Lim D-J, Ha S-K, Kim S-D, Kim S-H. Fatal case of cerebral aspergillosis: a case report and literature review. Journal of Korean Neurosurgical Society. 2012;52(4):420

5. Rasoolinejad M, Hajiabdolbaghi M, Dehghan Manshadi SA, Azmoudeh Ardalan F, Moradnejad P, Alijani N. Cerebral Aspergillosis in an Immunocompetent Patient: A Case Report. Arch Neurosci. [Case Report]. 2016;3(2):e31710.

6. Patterson TF, Thompson III GR, Denning DW, Fishman JA, Hadley S, Herbrecht R, et al. Practice guidelines for the diagnosis and management of aspergillosis: 2016 update by the Infectious Diseases Society of America. Clinical Infectious Diseases. 2016;63(4):e1-e60

7. Fasciano JW, Ripple MG, Suarez JI, Bhardwaj A. Central nervous system aspergillosis: a case report and literature review. Hospital Physician. 1999;35:63-70

8. Artico M, Pastore FS, Polosa M, Sherkat S, Neroni M. Intracerebral Aspergillus abscess: case report and review of the literature. Neurosurgical review. 1997;20(2):135-8

9. Chakrabarti A, Chatterjee SS, Das A, Shivaprakash M. Invasive aspergillosis in developing countries. Medical mycology. 2011;49(Supplement_1):S35-S47

10. Zhang S, Fu Q, Chen Q, Liang T-b. Isolated cerebral aspergillosis in an immunocompetent woman on treatment for bacterial infected necrotizing pancreatitis: A case report. Medicine. 2017;96(48):e8908

11. Quenum K, Rifi L, Boutarbouch M, Arkha Y, Derraz S, Ouahabi AE, et al. Surgical Managment Of Intracranial Invasive Aspergillosis In Immunocompetent Patients: Results From 3 Case Reports. Neurological Research and Therapy. 2017;1(4):20 